\title{
Prognostic value of the advanced lung cancer inflammation index in early-stage non-small cell lung cancer patients undergoing video-assisted thoracoscopic pulmonary resection
}

\author{
Yan Wang, Lin Lin, Yanli Ji, Xiaoli Mei, Chunlin Zhao, Yu Chen, Guowei Che \\ Department of Thoracic Surgery, West China Hospital, Sichuan University, Chengdu, 610041, China \\ Contributions: (I) Conception and design: G Che; (II) Administrative support: G Che; (III) Provision of study materials or patients: Y Wang, L Lin, Y \\ Ji; (IV) Collection and assembly of data: X Mei, C Zhao, Y Chen; (V) Data analysis and interpretation: Y Wang, L Lin; (VI) Manuscript writing: All \\ authors; (VII) Final approval of manuscript: All authors. \\ Correspondence to: Guowei Che. Department of Thoracic Surgery, West China Hospital, Sichuan University, Guoxuexiang No. 37, Chengdu 610041, \\ China. Email: hx_guowei_che@foxmail.com.
}

\begin{abstract}
Background: The aim of this study was to assess the prognostic value of the preoperative advanced lung cancer inflammation index (ALI) in early stage non-small lung cancer (NSCLC) patients who received videoassisted thoracoscopic surgery (VATS) pulmonary surgery as their only therapy.

Methods: We retrospectively reviewed the medical records of patients who were diagnosed with earlystage NSCLC and received a VATS pulmonary resection from January 2014 to June 2016 in the Department of Thoracic Surgery, West China Hospital. A receiver operating characteristic (ROC) curve analysis was conducted to determine the optimal cut-off values, and patients were divided into low and high ALI groups. The Kaplan-Meier method and Cox proportional hazards model were used to evaluate potential predictors of overall survival (OS) and disease-free survival (DFS).

Results: A total of 292 patients were enrolled in our analysis. Then, 155 and 137 patients were assigned to the low ALI (ALI <50) and high (ALI >50) groups, respectively. The multivariate analyses revealed that preoperative ALI $<50$ was an independent prognostic factor for both OS [hazard ratio (HR) $=2.603,95 \%$ confidence interval (95\% CI): 1.128-6.006, P=0.025] and DFS (HR =2.372, 95\% CI: 1.141-4.935, P=0.021), and patients with a low preoperative ALI had worse OS $(\mathrm{P}<0.001)$ and DFS $(\mathrm{P}<0.001)$ compared with those with a high preoperative ALI.

Conclusions: A low preoperative ALI was significantly correlated with poor survival, and the ALI might serve as a promising marker of prognosis in early-stage NSCLC patients who received a VATS pulmonary resection as their only therapy.
\end{abstract}

Keywords: Advanced lung cancer inflammation index; early stage non-small cell lung cancer; video-assisted thoracoscopic surgery (VATS); prognosis

Submitted Aug 17, 2019. Accepted for publication Feb 14, 2020.

doi: 10.21037/apm.2020.03.18

View this article at: http://dx.doi.org/10.21037/apm.2020.03.18

\section{Introduction}

Lung cancer remains the leading cause of tumor-related deaths worldwide, and non-small cell lung cancer (NSCLC) accounts for approximately $85 \%$ of all lung cancer cases $(1,2)$. With the advances in video-assisted thoracoscopic surgery (VATS), surgery has been the major treatment for early-stage NSCLC patients, and the prognosis of these patients has improved to a certain extent (3). However, to date, the tumor-node-metastasis (TNM) stage is still the most common indicator for predicting the survival of NSCLC patients. More valuable and reliable prognostic biomarkers are urgently needed. 
Inflammation status has been proven to play a key role in cancer development and long-term clinical outcomes of cancer patients (4-6). As previously reported, there are several inflammation biomarkers that have high prognostic value in NSCLC patients undergoing lung resection, including the neutrophil-lymphocyte ratio (NLR) (7), the platelet-lymphocyte ratio (PLR) (8), the lymphocytemonocyte ratio (LMR) (9) and C-reactive protein (CRP) (10). On the other hand, nutritional status has also been demonstrated to show a strong linkage with survival after surgery in NSCLC $(11,12)$. Tewari et al. (12) and Nakagawa et al. (13) showed that low body mass index (BMI), which represented impaired nutrition, was significantly associated with worse long-term survival in patients with resected NSCLC. Albumin (ALB) is also a promising indicator for nutritional status, and hypoalbuminemia is correlated with poor prognosis in patients with NSCLC (14). In 2013, Jafri et al. (15) developed a novel prognostic index named the advanced lung cancer inflammation index (ALI) and evaluated its value for prognosis in patients with metastatic NSCLC. The ALI consists of BMI, ALB and NLR, which incorporate both inflammatory and nutritional factors. Subsequently, its usefulness has been reported in malignant lymphoma (16), esophageal carcinoma (17), small cell lung cancer (SCLC) (18) and NSCLC (19). However, no study has focused on earlystage NSCLC patients who undergo VATS pulmonary operation as the only treatment.

Therefore, the aim of this study was to explore the prognostic value of preoperative ALI in patients who were diagnosed with limited-stage NSCLC and received only VATS pulmonary resection (either lobectomy or segmentectomy).

\section{Methods}

\section{Study design}

This was a retrospective single-center study.

\section{Patient selection}

Patients with NSCLC who underwent VATS pulmonary surgery from January 2014 to June 2016 in the Department of Thoracic Surgery, West China Hospital, Sichuan University, were reviewed retrospectively. The inclusion criteria were as follows: (I) pathologically diagnosed with NSCLC in TNM stage I or II; (II) VATS pulmonary resection with lymph node dissection was the only treatment; (III) available preoperative BMI, serum ALB and NLR values; (IV) adequate clinicopathologic and followup data; (V) no acute or chronic inflammatory diseases preoperatively, such as active infection or rheumatoid arthritis; (VI) normal liver function; and (VII) no second malignancies or recurrent NSCLC. Patients were excluded according to the exclusion criteria: (I) received neoadjuvant, postoperative adjuvant or immunological therapies; (II) intraoperative conversion to thoracotomy; or (III) concomitant hepatic or inflammatory diseases.

\section{Data collection}

All preoperative clinicopathological data were extracted from the medical records of our hospital. They included age, gender, BMI, smoking history, preoperative comorbidity, lung function, tumor location, size, pathology, type of resection, TNM stage, serum ALB, NLR and platelet-lymphocyte ratio (PLR) levels. BMI was calculated as weight in kilograms divided by height in square meters. NLR and PLR were defined as a simple ratio between the neutrophil and lymphocyte counts and a simple ratio between the platelet and lymphocyte counts, respectively. The ALI was calculated as BMI $\times$ ALB/NLR .

Overall survival (OS) was defined as the date of surgery to the date of death from any cause or last follow-up, and disease-free survival (DFS) was defined as the date of surgery to the date of local recurrence, distant metastasis or last follow-up. The follow-up time was from the date of surgery to September 2018 or the date of last followup. During the follow-up period, patients received routine follow-up tests, such as computed tomography (CT) scans, every two months or/and telephone contacts to assess the patient's current conditions.

\section{Statistical analysis}

The thresholds for BMI, ALB, NLR and ALI were determined by receiver operating characteristic (ROC) curve analyses for the prediction of death from any cause. The thresholds for other continuous variables were determined using their median values. The chi-square test or Fisher's exact test was performed to compare differences between ALI groups. Univariate and multivariate analyses were conducted using the Cox proportional hazard model to identify independent predictors for OS and DFS, and all variables with a $\mathrm{P}$ value $<0.10$ in the univariate analysis were 
Table 1 Baseline clinicopathological characteristics

\begin{tabular}{lc}
\hline Characteristics & Value \\
\hline Patients, $\mathrm{n}$ & 292 \\
Age (median, range), years & $62[18-81]$ \\
Male/Female & $158 / 134$ \\
BMI (median, range), kg/m & $23.48(15.62-32.27)$ \\
Smoking history, $\mathrm{n}$ & 134 \\
Preoperative comorbidity & \\
COPD & 39 \\
Emphysema & 44 \\
Tumor location (left/right) & $100 / 192$ \\
Tumor size (median, range), cm & $2.4(0.5-5.0)$ \\
Pathology (AC/SC/others) & $197 / 71 / 24$ \\
Extent of resection & $204 / 88$ \\
(lobectomy/segmentectomy) & \\
TNM stage (I/ll) & $197 / 95$ \\
\hline
\end{tabular}

BMI, body mass index; COPD, chronic obstructive pulmonary disease; AC, adenocarcinoma; SC, squamous carcinoma; TNM, tumor-node-metastasis.

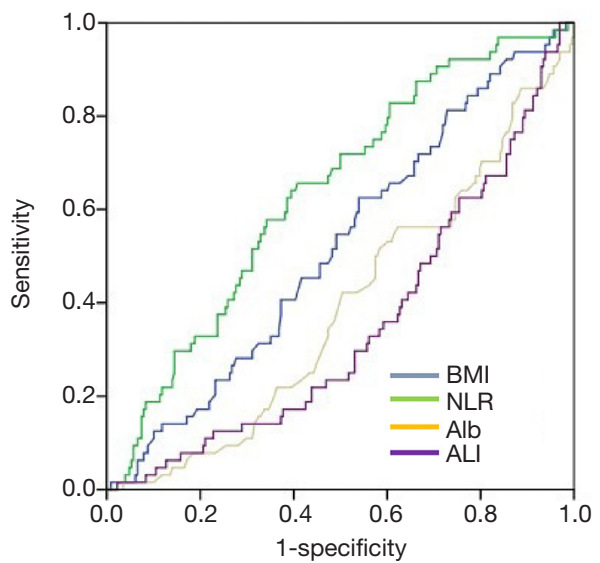

Figure 1 ROC curves for survival prediction (overall survival). BMI, body mass index; NLR, neutrophil to lymphocyte ratio; Alb, albumin; ALI, advanced lung cancer inflammation index; ROC, receiver operating characteristic.

included in the multivariate analysis. Survival curves were calculated by the Kaplan-Meier method, and differences between groups were analyzed by the log-rank test. All analyses were performed using the IBM SPSS statistics software program, version 22.0. A P value $<0.05$ was considered statistically significant.

\section{Results}

\section{Characteristics of the study population}

A total of 292 patients were included in the current research. The baseline clinicopathological characteristics of these patients are summarized in Table 1. The median age, BMI and tumor size were 62 years (range, 1881 years), $23.48 \mathrm{~kg} / \mathrm{m}^{2}\left(15.62-32.27 \mathrm{~kg} / \mathrm{m}^{2}\right)$ and $2.4 \mathrm{~cm}$ $(0.5-5.0 \mathrm{~cm})$, respectively. Of all cases, 158 patients were male, 134 patients were current or ex-smokers, 39 patients had chronic obstructive pulmonary disease, 44 patients had emphysema, 100 cases were located on the left, 197 cases were adenocarcinoma (AC), 204 patients received lobectomy and 197 patients were in TNM stage I.

\section{Cut-off values defined by ROC curve analyses}

The critical values of BMI, ALB, NLR and ALI were 24.79 (sensitivity $68.8 \%$ and specificity $35.1 \%$ ), 4.32 (sensitivity $76.0 \%$ and specificity $44.6 \%$ ), 1.67 (sensitivity $82.3 \%$ and specificity $41.6 \%$ ) and 50.0 (sensitivity $67.7 \%$ and specificity $64.5 \%$ ), respectively (Figure 1).

\section{Association between ALI and patient characteristics}

The correlation of ALI with the clinicopathological characteristics of patients is shown in Table 2. Patients in the low ALI group (ALI <50) had a significantly higher proportion of men $(\mathrm{P}=0.002)$, lower $\mathrm{BMI}(\mathrm{P}=0.004)$, a higher proportion of cigarette smoking history $(\mathrm{P}<0.001)$, lower forced vital capacity (FVC) $(\mathrm{P}=0.007)$, larger tumors $(\mathrm{P}=0.002)$, a lower proportion of $\mathrm{AC}(\mathrm{P}=0.032)$, higher NLR $(\mathrm{P}<0.001)$ and higher PLR $(\mathrm{P}<0.001)$.

\section{Predictive factors for OS}

The univariate analysis suggested that male sex $(\mathrm{P}=0.008)$, smoking history $(\mathrm{P}<0.001)$, larger tumors $(\mathrm{P}=0.018)$, TNM stage II $(\mathrm{P}=0.047)$, an ALB level $<4.32 \mathrm{~g} / \mathrm{dL}(\mathrm{P}=0.004)$, NLR $\geq 1.67(\mathrm{P}=0.001)$ and $\mathrm{ALI}<50(\mathrm{P}<0.001)$ were potential independent factors for OS. The multivariate analysis showed that only the ALI was significantly correlated with OS (HR $=2.603$, 95\% CI: 1.128-6.006, $\mathrm{P}=0.025$ ) (Table 3). Patients with an ALI $<50$ had a 
Table 2 Comparison of clinicopathological characteristics between low and high ALI groups

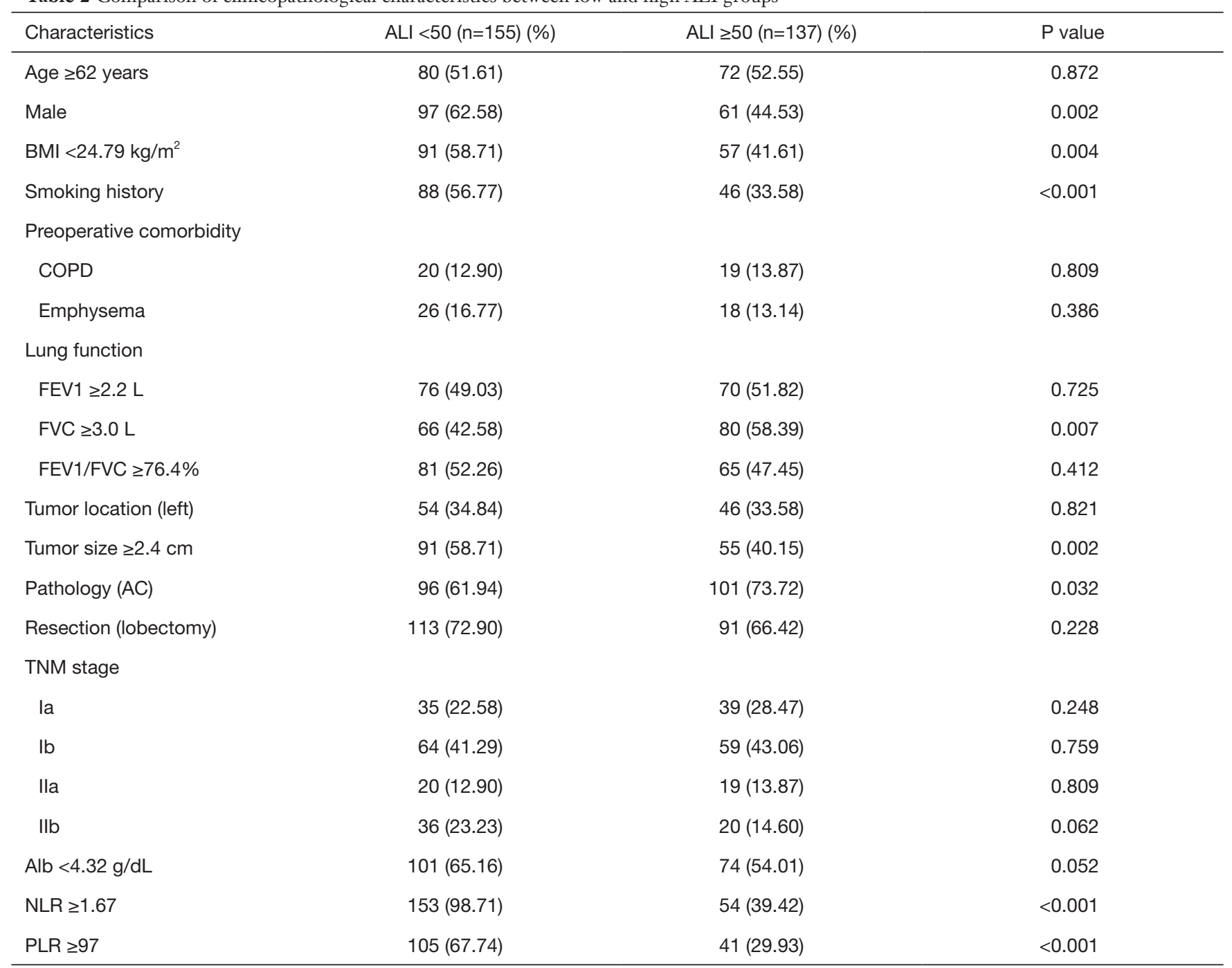

ALI, advanced lung cancer inflammation index; BMI, body mass index; COPD, chronic obstructive pulmonary disease; FEV1, forced expiratory volume in one second; FVC, forced vital capacity; AC, adenocarcinoma; TNM, tumor-node-metastasis; Alb, albumin; NLR, neutrophil to lymphocyte ratio; PLR, platelet to lymphocyte ratio.

significantly worse OS than patients with an ALI $\geq 50$ $(\mathrm{P}<0.001)$ (Figure 2).

\section{Predictive factors for DFS}

The univariate analysis revealed that smoking status $(\mathrm{P}=0.009)$, tumor size $(\mathrm{P}<0.001)$, TNM stage $(\mathrm{P}=0.037)$, NLR $(\mathrm{P}=0.009)$ and ALI values $(\mathrm{P}<0.001)$ were significantly associated with DFS. Tumor size $\geq 2.4 \mathrm{~cm}$ (HR $=1.860$, 95\% CI: $1.108-3.121, \mathrm{P}=0.019)$ and $\mathrm{ALI}<50(\mathrm{HR}=2.372$, 95\% CI: $1.141-4.935, \mathrm{P}=0.021)$ were demonstrated to be independent risk factors for poorer DFS (Table 4). Patients in the high ALI group had significantly better survival than patients in the low ALI group $(\mathrm{P}<0.001)$ (Figure 3).

\section{Discussion}

Nutrition and inflammation status are well known to be correlated with the prognosis of malignancies $(4-6,11,12)$. BMI and ALB level are valuable parameters for the evaluation of nutritional status. A decreased muscle and fat burden caused by cancer progression results in body weight loss and a higher risk of mortality (20). Although the detailed mechanism remains unclear, malnutrition 
Table 3 Univariate and multivariate Cox regression analyses to assess the prognostic factors for overall survival in all patients

\begin{tabular}{|c|c|c|c|c|}
\hline Characteristics & \multicolumn{2}{|c|}{ Univariate analysis } & \multicolumn{2}{|c|}{ Multivariate analysis } \\
\hline Age $\geq 62$ years & $1.566(0.950-2.584)$ & 0.079 & $1.338(0.787-2.275)$ & 0.282 \\
\hline Male & $2.018(1.205-3.378)$ & 0.008 & $0.729(0.266-1.994)$ & 0.538 \\
\hline $\mathrm{BMI}<24.79 \mathrm{~kg} / \mathrm{m}^{2}$ & $0.809(0.492-1.329)$ & 0.402 & & \\
\hline \multicolumn{5}{|l|}{ Preoperative comorbidity } \\
\hline COPD & $1.254(0.620-2.537)$ & 0.53 & & \\
\hline Emphysema & $1.689(0.933-3.057)$ & 0.083 & $1.371(0.722-2.603)$ & 0.335 \\
\hline \multicolumn{5}{|l|}{ Lung function } \\
\hline FEV1/FVC $\geq 76.4 \%$ & $1.632(0.990-2.691)$ & 0.055 & $1.120(0.659-1.905)$ & 0.675 \\
\hline Tumor location (left) & $1.236(0.752-2.032)$ & 0.402 & & \\
\hline Tumor size $\geq 2.4 \mathrm{~cm}$ & $1.833(1.112-3.022)$ & 0.018 & $1.195(0.697-2.046)$ & 0.518 \\
\hline Pathology (AC) & $0.962(0.659-1.406)$ & 0.843 & & \\
\hline Resection (lobectomy) & $1.501(0.843-2.673)$ & 0.168 & & \\
\hline TNM stage II & $1.644(1.006-2.687)$ & 0.047 & $1.411(0.834-2.387)$ & 0.199 \\
\hline Alb $<4.32$ g/dL & $2.331(1.308-4.152)$ & 0.004 & $1.717(0.941-3.134)$ & 0.078 \\
\hline$N L R \geq 1.67$ & $3.652(1.667-8.002)$ & 0.001 & $1.391(0.488-3.966)$ & 0.537 \\
\hline
\end{tabular}

BMI, body mass index; COPD, chronic obstructive pulmonary disease; FEV1, forced expiratory volume in one second; FVC, forced vital capacity; AC, adenocarcinoma; TNM, tumor-node-metastasis; Alb, albumin; NLR, neutrophil to lymphocyte ratio; PLR, platelet to lymphocyte ratio; ALI, advanced lung cancer inflammation index.

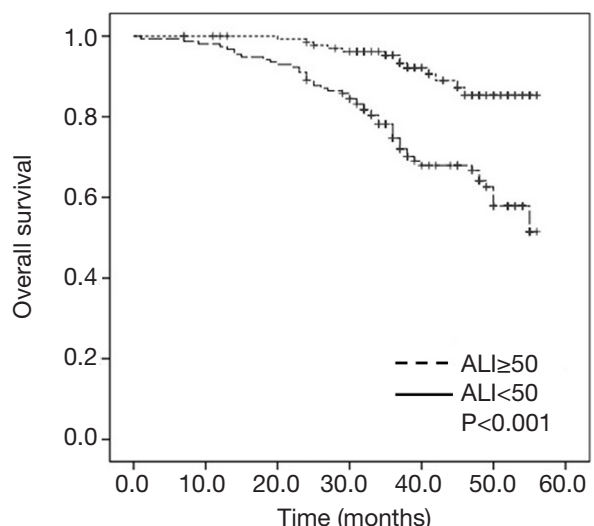

Figure 2 Kaplan-Meier overall survival curves stratified by ALI. ALI, advanced lung cancer inflammation index. may cause the deterioration of the immune system (19). However, the utility of BMI is usually limited due to its inaccurate representation of body compositions, and body weight loss is indistinct in patients with pleural effusion or body edema. Furthermore, the concentration of ALB can be affected by many factors, such as illness, dehydration and fluid retention $(21,22)$, which greatly restricts its utility in clinical practice.

Previous studies have demonstrated that lymphocytes play a crucial role in killing tumor cells and regulating apoptosis, angiogenesis, proliferation and metastasis of tumors by secreting cytokines $(11,23)$. On the other hand, neutrophils can inhibit lymphocyte-mediated immune activity consisting of activated $\mathrm{T}$ cells and natural killer $\mathrm{T}$ cells by producing 
Table 4 Univariate and multivariate Cox regression analyses to assess the prognostic factors for disease-free survival in all patients

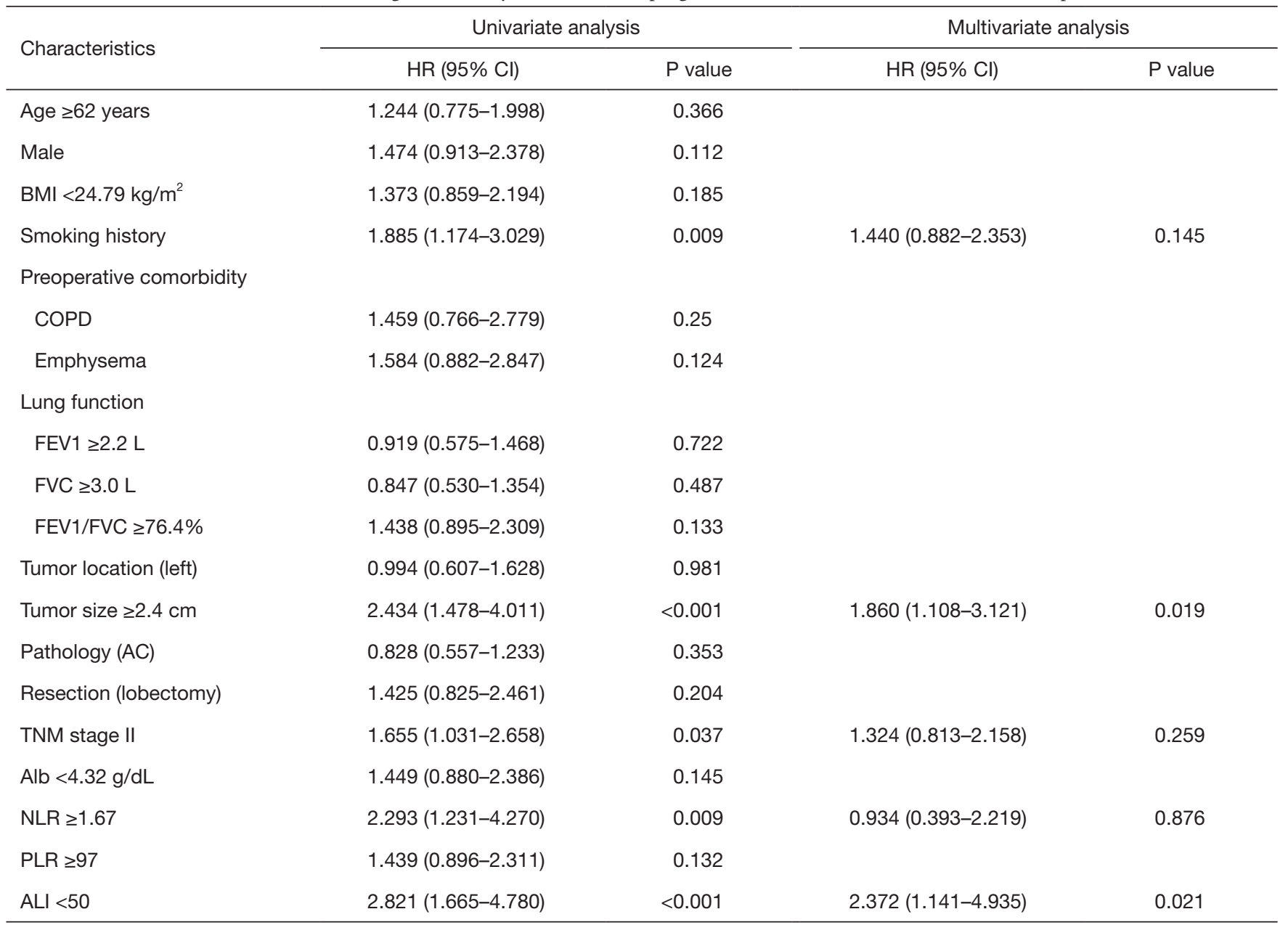

BMI, body mass index; COPD, chronic obstructive pulmonary disease; FEV1, forced expiratory volume in one second; FVC, forced vital capacity; AC, adenocarcinoma; TNM, tumor-node-metastasis; Alb, albumin; NLR, neutrophil to lymphocyte ratio; PLR, platelet to lymphocyte ratio; ALI, advanced lung cancer inflammation index.

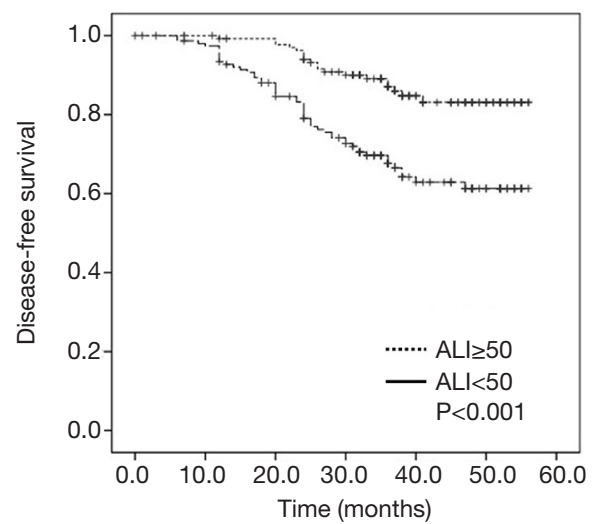

Figure 3 Kaplan-Meier disease-free survival curves stratified by ALI. ALI, advanced lung cancer inflammation index. cytokines (18,24). A meta-analysis including 7,219 patients reported that an elevated NLR was a negative predictor of survival in patients with lung cancer (25).

Nevertheless, a single marker or ratio often fails to assess the prognosis of patients precisely. In 2003, Forrest et al. (26) proposed the Glasgow prognostic score (GPS), which combined CRP and ALB levels, and a number of studies have reported its prognostic value in several cancers (26-30). Subsequently, McMillan et al. (31) proposed the modified Glasgow prognostic score (mGPS), which was more sensitive than the GPS, and a meta-analysis involving 5,817 patients revealed that it was a good predictor for the survival of lung cancer patients (32). Thus, a combination of 
multiple indexes may perform better than single biomarkers in predicting prognosis in patients with lung cancer. In 2013, Jafri et al. (15) described a novel index called the ALI, and several studies have proven its high prognostic value in patients with NSCLC $(18,19)$. However, none of them focused on early-stage patients who received VATS pulmonary resection as the only treatment. Therefore, we conducted this research to explore the association between preoperative ALI and prognosis in this population.

Compared with previous relevant studies, our research has several advantages or highlights. Only early stage (TNM I-II) patients were enrolled in the analysis. In addition, all of the included patients received the VATS pulmonary operation as their only treatment; as chemoradiotherapy and immunotherapy can significantly affect the inflammatory immune status, this significantly reduced the bias caused by therapy methods. Patients with hepatic diseases were excluded because ALB is produced by the liver and abnormal liver function may cause a decreased ALB level. No significant difference in the proportion of patients at different disease stages was observed between the high and low ALI groups; to some extent, this may indicate that the TNM stage had no effect on the prognostic role of ALI in our study. In addition, the endpoint outcomes in our research included OS and DFS, while most previous studies only reported OS. The cut-off value for the ALI was 50 in our study, which is much higher than the thresholds reported in previous studies including all-stage patients; meanwhile, compared with these previous studies, the median values of the ALB level and NLR of our study were significantly higher and lower, respectively. This indicates that the optimal critical value of ALI should be different for patients with different stages and that limited stage patients may have a relatively higher ALI threshold.

There are still some limitations that should be addressed. First, this was a retrospective single-center study with a small sample size. Second, there were some significant differences in the clinicopathological characteristics between the high and low ALI groups, and the influence of these factors on survival and the prognostic role of the ALI should not be ignored. Third, as mentioned above, the critical value of the ALI that maximized the Youden index in our study was 50 ; whether 50 is the optimal cutoff point still needs be tested in future studies. Fourth, the ALI lacks tumor specificity and does not include other important biomarkers, such as CRP, platelet-lymphocyte ratio (PLR), lymphocyte-monocyte ratio (LMR), etc.

In conclusion, our research demonstrated that the preoperative ALI may serve as a promising prognostic index in early-stage NSCLC patients who receive only pulmonary resection by VATS. More well-designed multicenter studies with larger samples are needed to verify our findings.

\section{Acknowledgments}

Funding: None.

\section{Footnote}

Conflicts of Interest: All authors have completed the ICMJE uniform disclosure form (available at http://dx.doi. org/10.21037/apm.2020.03.18). The authors have no conflicts of interest to declare.

Ethical Statement: The authors are accountable for all aspects of the work in ensuring that questions related to the accuracy or integrity of any part of the work are appropriately investigated and resolved. All procedures performed in studies involving human participants were in accordance with the ethical standards of the Ethics Committee of West China Hospital, Sichuan University, the national research committee and the 1964 Helsinki Declaration and its later amendments or comparable ethical standards. The study was approved by the regional committee of Sichuan University West China Hospital (ID: 2018-571). Written informed consent for the use and storage of information from the hospital database was obtained from each patient.

Open Access Statement: This is an Open Access article distributed in accordance with the Creative Commons Attribution-NonCommercial-NoDerivs 4.0 International License (CC BY-NC-ND 4.0), which permits the noncommercial replication and distribution of the article with the strict proviso that no changes or edits are made and the original work is properly cited (including links to both the formal publication through the relevant DOI and the license). See: https://creativecommons.org/licenses/by-nc-nd/4.0/.

\section{References}

1. Früh M, De Ruysscher D, Popat S, et al. Small-cell lung cancer (SCLC): ESMO Clinical Practice Guidelines for diagnosis, treatment and follow-up. Ann Oncol 2013;24 Suppl 6:vi99-105.

2. Torre LA, Bray F, Siegel RL, et al. Global cancer statistics, 
2012. CA Cancer J Clin 2015;65:87-108.

3. Siegel R, DeSantis C, Virgo K, et al. Cancer treatment and survivorship statistics, 2012. CA Cancer J Clin 2012;62:220-41.

4. Coussens LM, Werb Z. Inflammation and cancer. Nature 2002;420:860-7.

5. Grange JM, Krone B, Mastrangelo G. Infection, inflammation and cancer. Int J Cancer 2011;128:2240-1.

6. Proctor MJ, Morrison DS, Talwar D, et al. A comparison of inflammation-based prognostic scores in patients with cancer. A Glasgow Inflammation Outcome Study. Eur J Cancer 2011;47:2633-41.

7. Tomita M, Shimizu T, Ayabe T, et al. Elevated preoperative inflammatory markers based on neutrophiltolymphocyte ratio and C-reactive protein predict poor survival in resected non-small cell lung cancer. Anticancer Res 2012;32:3535-8.

8. Kim SH, Lee HW, Go SI, et al. Clinical significance of the preoperative platelet count and platelet-to lymphocyte ratio (PLT-PLR) in patients with surgically resected nonsmall cell lung cancer. Oncotarget 2016;7:36198-206

9. Xia H, Sun Z, Deng L, et al. Prognostic Significance of the Preoperative Lymphocyte to Monocyte Ratio in Patients with Stage I Non-Small Cell Lung Cancer Undergoing Complete Resection. Cancer Invest 2016;34:378-84.

10. Alifano M, Falcoz PE, Seegers V, et al. Preresection serum C-reactive protein measurement and survival among patients with resectable non-small cell lung cancer. J Thorac Cardiovasc Surg 2011;142:1161-7.

11. Eerola AK, Soini Y, Paakko P. Tumour infiltrating lymphocytes in relation to tumour angiogenesis, apoptosis and prognosis in patients with large cell lung carcinoma. Lung Cancer 1999;26:73-83.

12. Tewari N, Martin-Ucar AE, Black E, et al. Nutritional status affects long term survival after lobectomy for lung cancer. Lung Cancer 2007;57:389-94.

13. Nakagawa T, Toyazaki T, Chiba N, et al. Prognostic value of body mass index and change in body weight in postoperative outcomes of lung cancer surgery. Interact CardioVasc Thorac Surg 2016;23:560-6.

14. Miyoshi T, Misumi N, Hiraike M, et al. Risk Factors Associated with Cisplatin-Induced Nephrotoxicity in Patients with Advanced Lung Cancer. Biol Pharm Bull 2016;39:2009-14.

15. Jafri SH, Shi R, Mills G. Advance lung cancer inflammation index (ALI) at diagnosis is a prognostic marker in patients with metastatic non-small cell lung cancer (NSCLC): a retrospective review. BMC Cancer
2013;13:158.

16. Park YH, Yi HG, Lee MH, et al. Prognostic value of the pretreatment advanced lung cancer inflammation index (ALI) in diffuse large B cell lymphoma patients treated with R-CHOP chemotherapy. Acta Haematol 2017;137:76-85.

17. Feng JF, Huang Y, Chen QX. A new inflammation index is useful for patients with esophageal squamous cell carcinoma. Onco Targets Ther 2014;7:1811-5.

18. He X, Zhou T, Yang Y, et al. Advanced lung cancer inflammation index, a new prognostic score, predicts outcome in patients with small-cell lung cancer. Clin Lung Cancer 2015;16:e165-71.

19. Tomita M, Ayabe T, Nakamura K, et al. The advanced lung cancer inflammation index is an independent prognostic factor after surgical resection in patients with non-small-cell lung cancer. Interact Cardiovasc Thorac Surg 2018;26:288-92.

20. McMillan DC. An inflammation-based prognostic score and its role in the nutrition-based management of patients with cancer. Proc Nutr Soc 2008;67:257-62.

21. Yao Y, Zhao M, Yuan D, et al, Elevated pretreatment serum globulin albumin ratio predicts poor prognosis for advanced non-small cell lung cancer patients. J Thorac Dis 2014;6:1261-70.

22. Duran AO, Inanc M, Karaca H, et al, Albumin-globulin Ratio for Prediction of Long-term Mortality in Lung Adenocarcinoma Patients. Asian Pac J Cancer Prev 2014;15:6449-53.

23. Eerola AK, Soini Y, Paakko P. A high number of tumorinfiltrating lymphocytes are associated with a small tumor size, low tumor stage, and a favorable prognosis in operated small cell lung carcinoma. Clin Cancer Res 2000;6:1875-81.

24. Kim EY, Kim N, Kim YS, et al. Prognostic significance of modified advanced lung cancer inflammation index (ALI) in patients with small cell lung cancer comparison with original ALI. PLoS One 2016;11:e0164056.

25. Yu Y, Qian L, Cui J. Value of neutrophil-to-lymphocyte ratio for predicting lung cancer prognosis: A meta-analysis of 7,219 patients. Mol Clin Oncol 2017;7:498-506.

26. Forrest LM, McMillan DC, McArdle CS, et al. Evaluation of cumulative prognostic scores based on the systemic inflammatory response in patients with inoperable nonsmall-cell lung cancer. Br J Cancer 2003;89:1028-30.

27. Nozoe T, Iguchi T, Egashira A, et al. Significance of modified Glasgow prognostic score as a useful indicator for prognosis of patients with gastric carcinoma. Am J Surg 
2011;201:186-91.

28. Ishizuka M, Kubota K, Kita J, et al. Impact of an inflammation-based prognostic system on patients undergoing surgery for hepatocellular carcinoma: a retrospective study of 398 Japanese patients. Am J Surg 2012;203:101-6.

29. Ishizuka M, Nagata H, Takagi K, et al. Inflammationbased prognostic score is a novel predictor of postoperative outcome in patients with colorectal cancer. Ann Surg 2007;246:1047-51.

Cite this article as: Wang Y, Lin L, Ji Y, Mei X, Zhao C, Chen Y, Che G. Prognostic value of the advanced lung cancer inflammation index in early-stage non-small cell lung cancer patients undergoing video-assisted thoracoscopic pulmonary resection. Ann Palliat Med 2020;9(3):721-729. doi: 10.21037/ apm.2020.03.18
30. Vashist YK, Loos J, Dedow J, et al. Glasgow Prognostic Score is a predictor of perioperative and long-term outcome in patients with only surgically treated esophageal cancer. Ann Surg Oncol 2011;18:1130-8.

31. McMillan DC. The systemic inflammation-based Glasgow Prognostic Score: a decade of experience in patients with cancer. Cancer Treat Rev 2013;39:534-40.

32. Jin J, Hu K, Zhou Y, et al. Clinical utility of the modified Glasgow prognostic score in lung cancer: A metaanalysis. PLoS One 2017;12:e0184412. 